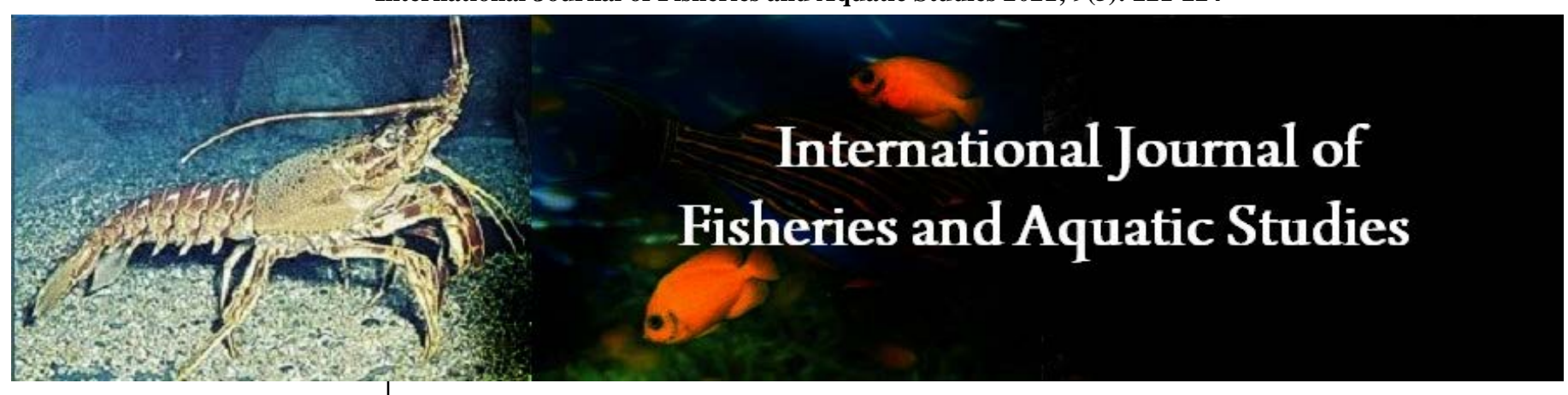

E-ISSN: 2347-5129

P-ISSN: 2394-0506

(ICV-Poland) Impact Value: 5.62

(GIF) Impact Factor: 0.549

IJFAS 2021; 9(5): 221-224

(C) 2021 IJFAS

www.fisheriesjournal.com

Received: 13-07-2021

Accepted: 19-08-2021

Dr. Sangeeta Singh

Department of Zoology, Bareilly College, Bareilly, Uttar Pradesh, India

Dr. Kshama Dwivedi

Department of Zoology, Bareilly College, Bareilly, Uttar Pradesh, India
Corresponding Author: Dr. Sangeeta Singh Department of Zoology, Bareilly College, Bareilly, Uttar Pradesh, India

\section{Gross variations in relative size of brain and its subdivisions among fishes: An overview}

\author{
Dr. Sangeeta Singh and Dr. Kshama Dwivedi
}

DOI: https://doi.org/10.22271/fish.2021.v9.i5c.2608

\begin{abstract}
Fishes are the aquatic vertebrates inhabiting all the levels of the water column. Different species of fishes are well adapted to successfully live in all types of water bodies. They possess a well-developed nervous system to combat all the challenges they encounter in their specified habitat in the water column. Fishes that are migratory in nature are also able to overcome the problems faced by them in moving from one type of water body to the other. Although the sub divisions of the brain in different species of fish are same, there occurs a considerable variation among them owing to the whole brain size, shape and size of sub divisions etc. Studies have reported variations in whole brain size and enlargement of a particular brain subdivision that may be correlated with the body size, ecological niche or habitat of the species or cognitive ability of the species. Most of the studies performed with this objective reported interspecific variations among fishes. Fewer studies however, reported intraspecific morphological variations in brain/brain subdivisions among the fish population. Nevertheless, brains are energetically costly organs. Super class Pisces including all the fishes is a diverse group with respect to body size and habitat within water column and all such studies are so scattered that it is difficult to summarize them all. Therefore, the aim of this paper is to discuss and provide information about the variations in the brain size and in its subdivisions and unique brain structures found in fishes. Such studies were conducted in earlier times also and are gaining a great interest since past 10 years.
\end{abstract}

Keywords: subdivisions, pisces, brain

\section{Introduction}

Fishes are the first jawed aquatic vertebrates characterized by a stream lined body and the presence of paired and unpaired fins and gills. They possess a well-developed nervous system characterized by the presence of brain, spinal cord and nerves. Brain is the most mysterious organ with complex structure and functions and both common man and scientists have keen interest in gaining knowledge about this organ. The primitive jawless vertebrates, lampreys and hagfishes have small and relatively simple brain as compared to the jawed vertebrates (Iwanuik, 2017) ${ }^{[6]}$. Fish brains are typically small relative to its body size and are known to function to coordinate the activities of the body. Early in development, the brain of all craniates exists as a simple tube derived from the ectodermal layer which soon gets differentiated into three vesicles, prosencephalon, mesencephalon and rhombencephalon that form forebrain, mid brain and hind brain respectively in the adult. Before discussing the variations in the whole brain size and subdivisions of fish brain, let us bring forward the gross morphological structure/features of fish brain in brief. In general, fish forebrain is differentiated into telencephalon and diencephalon, midbrain comprises of dorsal optic lobes and ventral tegmentum and hind brain gets differentiated into cerebellum and medulla oblongata.

Telencephalon is made up of a pair of olfactory lobes and two cerebral hemispheres. Olfactory lobes are concerned with the sense of smell. Diencephalon is a diamond shaped area that lies between the cerebral hemisphere and the optic lobe. The rostro-dorsal part of the rhombencephalon grows out to form the cerebellum and the dorsal part of the mesencephalon is differentiated into bilateral lobes which together form the tectum mesencephalon. The mid brain is thus, composed of a dorsal optic tectum which forms two optic lobes and a ventral tegmentum. Hind brain includes cerebellum and medulla oblongata. 
Only a small part of cerebellum can be seen from outside as corpus cerebella. Medulla oblongata is differentiated into one median facial lobe and two lateral vagal lobes. The number of these subdivisions is same in almost all the fish species. Broadly, the super class Pisces is a taxon group comprised of two major classes of living species namely Chondricthyes (cartilaginous fishes) and Osteicthyes (bony fishes). Chondrichthyes include the species that live in the marine environment and is divided into two subclasses- Selachii (sharks and rays) and Brachyodonti (Chimaera) while Osteicthyes is further divided into two subclassesActinopterygi (ray finned fishes) and Sarcopterygii (lobe finned fishes). These two subclasses are further grouped into many orders and suborders. Teleostei is one of the super orders of subclass Actinoptergii. Most of the studies deal with the morphological features of fish brain in teleost.

Various studies have been conducted to study of gross morphology and structure of brain in different fish species. The study of brain morphology is necessary as it has functional implications. However, such studies either deal with one or two fishes or are so disperse that it is difficult to have an overall view on the structural/morphological variations in fish brain. Therefore, the aim of this review is to summarize and discuss the principal knowledge regarding the structural modifications or morphology of fish brain subdivisions which may be related to different challenges a fish may face or concerned with the function and behavior of the fish or habitat.

\section{Whole Brain Size}

Various methods have been employed to measure the whole brain size that range from allometric measurements to analysis of brain size and brain region by using software. A large portion of the variation in brain features across vertebrate taxa is represented by variability in whole brain size (Iwaniuk, 2017) ${ }^{[6]}$. Though brain size tend to change with increase in body size, not all the variations in brain size is due to the allometric relationship as some species have a larger or smaller brain than other species of the same body size (Iwaniuk, 2017) ${ }^{[6]}$. It is important to study brain size variation in a species as it may have potential functional links to behavioural and cognitive performance of the organism. Increase in the brain size is due to the increase in the number of nerve cells and connections between neurons, whole brain size is thought to reflect general cognitive ability. Additionally, larger brains are associated with habitat spatial complexity (Shunway, 2008) ${ }^{[20]}$. In other words, variation in brain size may reflect the functional relationships between brain morphology and habitat. Brain size may also provide the idea of the environment a fish is living in or the challenges faced or encountered by the fish or vice versa. Sharks have much larger brain relative to body size than teleosts (Bauchot et al., 1976; Northcutt, 1978 and Lisney and Collin, 2006) ${ }^{[15,}$ 17, 11]. Apart from inter-specific variations in fishes, intraspecific large variations in terms of brain size and in the size of separate brain parts have also been reported in fishes. Variation in absolute and relative brain size differed significantly when compared among the population of wild sticklebacks (Pungitius pungitius) inhabiting in pond water and marine water (Gonda et al., 2011) ${ }^{[3]}$. Absolute and relative brain size was found to be larger in pond water populations than those of the marine water while telencephalon was found to be comparatively larger in marine population. All brain parts were found to be smaller when developed under laboratory conditions than in the wild, irrespective of population origin.

The environment conditions are known to have a significant role in the structural and functional development of the brain in organisms studied. Behavioral responses such as territoriality, parental care etc is correlated with the brain size; larger brain size in animal may lead to well-developed behavioural responses (Kotrschal et al., 1998) ${ }^{[7]}$. Species from different habitat and living under different environmental conditions show differences in brain sizes these variations in brain size may be correlated with the cognitive ability of a species.

\section{Brain Subdivisions}

Although the number of the major brain subdivision remains same, considerable interspecific variation/ differences do occur in the form and enlargement of different parts of the brain in fishes. Brains are energetically costly organs. Forebrain is the anterior most part of the brain and hence the name forebrain and is differentiated into two parts namely telencephalon and diencephalon. Telencephalon is composed of two parts: the anterior paired olfactory bulb/lobes and two large cerebral hemispheres posteriorly. In Elasmobranchs with relatively larger brains, the size of the telencephalon along with the cerebellum enlarge disproportionately while those parts of the brain that contain more direct sensory and motor connections do not show enlargement. The volume of telencephalon was found to significantly large in the sharks (Lisney and Collin, 2006) ${ }^{[11]}$. Larger telencephalon is found in fishes that are associated with higher utilization of near shore (Gonzalez-Voyer and Kolm, 2010) ${ }^{[4]}$. McCallium et al., 2014 found larger telencephalon in fish prior to spawning indicating requirement of increased cognitive demands of spawning.

Relative larger telencephalon size during the reproductive season was also reported in a wild fish, Neogobius melanostomus. Unlike the seasonal changes in tele mcephalon, changes in size other brain subdivisions were limited to a decrease in spring. Cerebral hemispheres, in the brain of actinopterygian fishes are solid joined with each other in the midline. In other words, lateral ventricles surrounded by nervous tissues are entirely lacking (Nieuwenhugs, 2009) ${ }^{[14]}$. Instead, they possess a single median ventral ventricle in contrast to the paired ventral (basal) ventricles in lower fishes.

Olfactory lobes/ Olfactory bulbs - The olfactory bulbs are the bilateral evaginations of the telencephalic vesicles which receive the primary sensory projections from the olfactory nerve and are responsible for olfaction. Olfactory bulb varies greatly among and within vertebrates (Iwaniuk, 2017) ${ }^{[6]}$. They show considerable variations in structure/size among species of fishes depending upon the feeding and social/reproductive behavior (Laberge and Hara, 2001) ${ }^{[9]}$. Elasmobranchs (sharks and dogfishes) or some bony fishes that depend mainly on smell for searching the food or social activities have remarkably enlarged olfactory lobes.

Fish olfaction takes place entirely in the aquatic environment where carrier of stimulant molecules water instead of air. Deep sea cartilaginous fishes have relatively large olfactory bulbs, whereas sharks and rays living in or near coral reefs have relatively small olfactory bulbs (Yopak, 2015) ${ }^{[24]}$. Benthic shark species display more developed olfactory bulbs than pelagic species (Krusha, 1988) ${ }^{[8]}$. They are also enlarged in many bottom feeding bony fishes compared with those in 
upper layers of water of the water column (Kotrschal el al., 1998; Gonzalez-Voyer and Kolm, 2010) ${ }^{[7,4]}$.

Enlarged olfactory bulbs are likely to aid the fish in navigation and/or predation (Yopak et al., 2015) ${ }^{[24]}$. Studies have shown that variability in olfactory bulb size among species of cartilaginous fishes is due to the variability in ecological niches or habitat occupied by the species or where vision is compromised and are independent of the allometric scaling from the rest of the brain. The relative largest olfactory bulbs were found in pelagic coastal /oceanic sharks, especially migratory species such as Carcharodon carcharias and Galeocerdo cuvier. In contrast, the smallest olfactory bulbs were found in the majority of reef associated species, including sharks from the families Carcharrhinidae and Hemiscyllidae and dasyatd batoids. The sense of smell plays an important role in fishes, mediating behaviours and physiological responses related to food search and feeding social interaction and many more (Sorensen and Caprio, 1998; Bone and Moore, 2008) [21, 2]

Diencephalon is covered dorsally by the posterior part of the cerebral hemisphere; its small area is visible between cerebral hemispheres and optic lobes. In bony fishes, brain is more specialized than elasmobranchs. They possess smaller cerebral hemispheres and diencephalon when compared to sharks. The saccus vasculosis is highly vascular protrusion of the ventral wall of the diencephalon of fishes. It is regarded as benthic organ detecting changes in water pressure.

Mesencephalon in fishes is made up of the dorsal optic tectum and the ventral tegmentum. In fish, reptiles and birds, optic tectum is the main visual processor of the brain and forms the roof. It is present in all the species of fish but it is developed differently in them. In teleosts, tectum is highly developed and plays an important role in taking rapid decisions required for the survival and reproduction and behavioural responses. It appears as two lobes from the dorsal side Tectum varies in size and it is relatively very large in fishes that feed by sight like Salmo. Tectum is about $0.8 \mathrm{~mm}$ thick with layered appearance in highly visual teleosts. It is analogous to the superior colliculus of mammals and also referred to as tectum mesencephali. In the brain of sunfish, the two lobes of the optic tectum are present between the forebrain and cerebellum and are connected together along the mid line by tectal commissure. In elasmobranchs, tectum not only attains smaller size than that of a typical teleost but also is less differentiated structure in relation to the cell types and layers. Very less data is available regarding the interspecific variation in the relative size of the components of the mesencephalon in these fishes.

Rhombencephalon constitutes the most caudal part of the brain, medulla oblongata. Rostrally, it borders on the mesencephalon and caudally it grades into the spinal cord. The cerebellum is a derivative of the rostral part of the rhombencephalon. Cerebellum is well developed in the cartilaginous fishes and is involved in various sensory-motor functions (Montgomery et al., 2012) ${ }^{[13]}$. The dogfish (small shark) possess simple bi-lobed cerebellum. However, the mackerel sharks which are larger in size and swifter have larger cerebellum occupying much part of the brain. In these fishes the cerebellum becomes convoluted to increase the surface area. The size of the cerebellum in Cetorhinus maximus (basking shark) is significantly large and accounts to the $30 \%$ of the total brain (Kruska, 1988) ${ }^{[8]}$. The cavity of the cerebellum or metacoel is prominent in sharks and rays and is completely disappeared in cerebellum of higher bony fishes

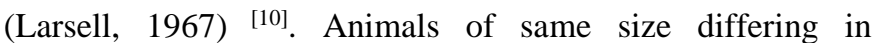
cerebellar complexity are seen among sharks (Northcutt, 1977) ${ }^{[16]}$.

Cerebellum of teleosts fish may vary in size and topography depending upon the relative development of the tracts bringing information to the cerebellum (Schnitzlein, 1964) [19]. Teleosts cerebellum is reported to play an important role in the execution of movements during swimming, controlling vestibulo-ocular reflex and emotional learning. The cerebellum and optic lobes are larger in bony fishes due to swimming habit. Fishes having a well-developed system of electroreception possess giant sized cerebellum which may larger than the rest of the brain. A significant reduction in the length of cerebellum and total brain length was observed in hatchery reared fish, Tor putitora when compared to that of their wild counter parts with no change in the telencephalon measurements. This change is attributed to the changed rearing environment, however the main factors behind it are not known.

Cerebellum of teleosts is subdivided into three parts namelycorpus cerebella, valvula cerebella and vestibule-lateral lobe. All these three parts are of the cerebellum vary considerably in size and shape. The valvula cerebelli is a unique specialization present only in actinopterygian fish. Valvula is very small in the fish, Amia but very large in the mormyrid, Gnathonemus covering the anterior part of the brain (forebrain). It is believed that the large size of the valvula in the mormyrid is correlated with the strong development of the electro-sensory system. Later, this view was ruled out. Fishes having well developed gustatory (carp) and lateral line (catfish) system have more developed valvula cerebella.

Corpus cerebelli seems to have no medially situated vermal part as found in that of the mammalian cerebellum. The small corpus cerebelli of the catfish are commonly considered to be homologus to only the vermal region of the mammalian corpus cerebella. Moreover, the cerebellum is with regard to both size and shape, by far the most variable part of the brain of actinopterygian fishes. In relation to cerebellum, corpus cerebelli and vestibule lateral cerebelli together make up about $42 \%$ of the brain in elasmobranch, Apristurus sinensis (South China catshark). The brain of Rhincodon typus possess highly foliated cerebellum, being the largest cerebellum within the chondrichthyes clade (Yopak and Frank, 2009) ${ }^{[22]}$. The different parts of medulla are enlarged with the development of various senses. Some fishes such as Culupea and Mugil possess prominent paired swelling called cristae cerebella, present on the anterolateral boundary of the fourth ventricles. These cristae are associated with schooling behavior of fishes. In goldfish, prominent vagal lobes are present behind the cristae cerebella.

There are certain structures found particularly in fishes which do need a mention. The presence of a structure named torus longitudinalis in the brain is a characteristic feature of teleosts. The mid ventral part of the optic tectum contains a protuberance called torus longitudinalis, which helps in integration between the sense of equilibrium and sense of vision. It is believed to compensate the absence of a true optic chiasma of bony fishes. Cartilaginous fishes are found to possess two structures in the dorsolateral wall of cerebellum which is similar to cerebellum but do not have climbing fibers. Hence, these two structures are referred to as cerebellum like structures and include medial and dorsal octavolateralis nuclei (MON and DON) respectively (Montgomery et al., 2012) ${ }^{[13]}$. Interestingly, such structures 
are also found in lampreys that belong to the jawless group Agnatha, sister clade to the jawed vertebrates. Moreover, a true cerebellum seems to be absent in the lampreys (Larsell, 1967; Northcutt, 2002) ${ }^{[10,18]}$. Absence of climbing fibers in this cerebellum like structure clearly indicates their role as sensory structures rather than motor structures.

In fish, Harriotta raleighana, both cerebellum and cerebellum like structures are very large and both together constitute 57 \% part of the brain (Yopak and Montgomery, 2008) ${ }^{\text {[23]. }}$ Comparative studies of brain size variations among mammalian and avian species have been reviewed by Healy and Rowe in $2007^{[5]}$ out of which most of the studies have been done in birds.

\section{Conclusions}

Fish species do show size variations in both whole brain size and brain subdivisions which may depend on many factors like habitat, ecological niche and cognitive ability of the species. It is time to conduct such studies in more species of fish which are not covered yet. Moreover, fish inhabit all the levels of water column facing many challenges to defend themselves from predators and other problems. As brains are energetically costly organ, enlargement of brain/subdivision will be favoured in accordance with the cognitive ability of the species and necessity of survival of a species. Most of the studies dealt with the variations either in the size of olfactory bulbs or that of cerebellum as compared to other brain structures.

The underlying mechanisms and the information about the environment clues or signals instigating the change in brain size are not clearly known till date. We do require using new/specialized techniques to precisely define and measure the brain size variations and especially in a particular subdivision of the brain of a species.

\section{Reference}

1. Axelrod CJ, Laberge F, Robinson BW. Intraspecific brain size variation between co-existing sunfish ecotypes. Proc. R. Soc. B 285: 2018, 1971. http://dx.doi.org/10.1098/rspb.2018.1971

2. Bone Q, Moore R. Biology of fishes. Oxford: Taylor and Francis 2008 [Google Scholar].

3. Gonda A, Herczeg G, Merila J. Population variation in brain size of nine-spined sticklebacks (Pungitius pungitius)- local adaptation or environmentally induced variation? Evol. Biol 2011;11:75

4. Gonzalez-Voyer A, Kolm N Sex. ecology and the brain evolutionary correlates of brain structure Volumes in Tanganyikan Cichlids. Plos ONE 2010;5(12):e14355. Doi:10.1371/journal.pone.0014355

5. Healy SD, Rowe C. A critique of comparative studies of brain size. Proc. R. Soc. B 2007;274:453-464 doi:10.1098/rspb.2006.3748

6. Iwanuik AN. The evolution of the nervous system in nonmammalian vertebrates. In Evolution of nervous system (second edition) Ed. Jon H. Kass 2017.

7. Kotrschal K, van Staaden MJ, Huber R. Fish brains: evolution and environmental relationships Rev Fish Bio. 1998, 373-408.

8. Krusha DCT. The brain of the basking shark (Cetorhinus maximus) Brain Behav. Evol 1988;32:353-363.

9. Laberge F, Hara TJ. Neurobiology of fish olfaction: a review. Brain Res Rev 2001;36(1):46.

10. Larsell $\mathrm{O}$. The comparative anatomy and histology of the cerebellum from myxonoids through birds. The University of Minnescota Press, Minnoapolis, 1967.

11. Lisney TJ, Collin SP. Brain morphology in large pelagic fishes: A comparison between sharks and teleosts. Journal of Fish Biology 2006;68(2):532-554.

12. McCallum ES, Capelle PM, Balshine S. Seasonal plasticity in telencephalon mass of a benthic fish. Journal of Fish Biology 2014;85(5):1785-1792.

13. Montgomery JC, Bodznick D, Yopak, KE. The cerebellum and cerebellum-like structures of Cartilaginous Fishes. Brain Behav Evol 2012;80:152165.

14. Nieuwenhugs R. The forebrain of actinopterygians revisited. Brain Behav. Evol 2009;73(4):229-252.

15. Bauchot R, Platel R, Ridet JM. Brain-body weight relationships in Selachii 1976;2:305-310.

16. Northcutt RG. Elasmobranch central nervous system organization and its possible evolutionary significance. Amer. Zool. 1977;17:411-429.

17. Northcutt RG. Brain organization in the cartilaginous fishes: In Hodgson ESand Mathewson RF (eds.) Sensory Biology of sharks, skates and rays 1978, 117-194.

18. Northcutt RG. Understanding vertebrate Brain evolution. In Integrative and Comparative Biology 2002;42(4):743759.

19. Schnitzlein HN. Correlation of habit and structure in the fish brain. Am. Zoologist 1964;4:21-32.

20. Shunway CA. Habitat complexity, brain and behaviour. Brain Behav, Evol 2008;72:123-124.

21. Sorenson PW, Caprio. J Chemoreception in fish. In R. E. Evans (Ed.) The physiology of Fishes. CRC press 1998, 375-406.

22. Yopak KE, Frank LR. Brain Size and brain organization of the Whale Shark, Rhincodon typus, using magnetic resonance imaging. Brain Behav Evol 2009;74:121-142.

23. Yopak KE, Montgomery JC. Brain organization and specialization in deep sea chondrichthyans. Brain Behav. Evol 2008;71:287-304.

24. Yopak KE, Lsiney TJ, Collin SP. Not all sharks are 'swimming noses': Variation in olfactory bulb size in cartilaginous fishes. Brain Struct. Funct 2015;220(2):1127-43. 